\title{
A Review of Envelope Tracking Power Supply for Mobile Communication Systems
}

\author{
Xinbo Ruan, Yazhou Wang, and Qian Jin
}

\begin{abstract}
In modern mobile communication systems, spectral efficient modulation formats have been widely used, which results in the envelope of the radio frequency (RF) signal is variable and having large peak to average power ratio (PAPR). In order to amplify the RF signal without distortion, the linear power amplifier (LPA) is adopted, which is less efficient when powered by constant voltage. Three popular techniques, i.e., Doherty, envelope elimination and restoration (EER), and envelope tracking (ET) techniques, to greatly improve the efficiency of the power amplifiers are analyzed and compared in this paper, and it is shown that the ET technique is the most suitable for future mobile communication systems. In the ET systems, the ET power supply is the key equipment, which dominates the system efficiency. With the development of the mobile communication systems, the bandwidth and the PAPR of the envelope signals are increasing rapidly, which pose severe challenges on the design of the ET power supply. This paper summarizes and sorts the ET power supplies in the literatures, and a detailed comparison is presented to guide the selection of ET power supplies for different applications. Finally, the methods, including soft-switching, slow envelope, and band separation are proposed for further increasing the efficiency of the ET power supply.
\end{abstract}

Index Terms - Band separation, Doherty, envelope elimination and restoration, envelope tracking, power amplifier, slow envelope, supply modulator, soft-switching.

\section{INTRODUCTION}

$\mathrm{T}$ HE mobile communication brings convenience of real-time communicating to people in a mobile state without physical transmission line, and it has been continuously developing ever since its advent in 1970s [1]. The first-generation $(1 \mathrm{G})$ mobile communication systems adopted analog electronics technology and can only provide voice service. Furthermore, it suffered poor anti-interference ability and slow data transmission rate. The second-generation $(2 \mathrm{G})$ mobile communication systems employed digital electronics technology, and they can transmit and receive voice and text message. With the rapidly increased customers and the demand for multi-media service, the data transmission rate is required to be faster, and the third-generation $(3 \mathrm{G})$ and fourth-generation $(4 \mathrm{G})$ mobile communication systems emerged in succession, which makes the video calling service and internet surfing into reality [1].

Manuscript received November 8, 2017. This work was supported by National Natural Science Foundation of China under Grant 51577090.

The authors are with the Center for More-Electric-Aircraft Power System, Nanjing University of Aeronautics and Astronautics, Nanjing, Jiangsu Province, China (e-mail: ruanxb@nuaa.edu.cn).

Digital Object Identifier 10.24295/CPSSTPEA.2017.00026

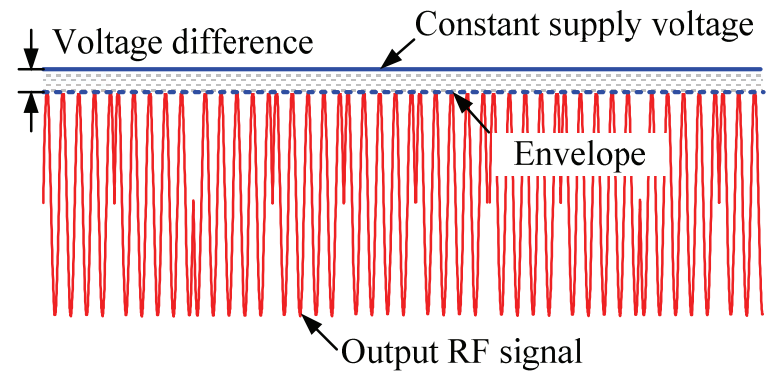

Fig. 1. Key waveforms of the LPA in $2 \mathrm{G}$ mobile communication system.

With the development of mobile communication, the power consumption increases rapidly, accounting for about $10 \%$ of the global power generation. Thus, it is necessary to reduce the power consumption of the mobile communication. In mobile communication systems, the base stations consume the majority of the power. In a base station, about $50 \%$ of the power is consumed by the power amplifier, and its loss is dissipated as heat, which requires extra power to cool down. So, increasing the efficiency of the power amplifier is the basis of reducing the overall power consumption of the mobile communication.

In $2 \mathrm{G}$ mobile communication systems, the modulation formats like Gaussian minimum shift keying (GMSK) and frequency shift keying (FSK) are employed [2]. Such modulation formats only modulate the phase and frequency of the radio frequency (RF) signal, and the amplitude of the RF signal does not carry any information. Thus, the envelope of the RF signal is constant, as shown in Fig. 1. Such signals can be amplified with nonlinear power amplifier (NLPA) or linear power amplifier (LPA). When LPA is adopted, the voltage difference between the supply voltage and the output RF signal of the LPA can be very small, as shown in Fig. 1, and the LPA can achieve relatively high efficiency [3].

In $3 \mathrm{G}$ and $4 \mathrm{G}$ mobile communication systems, for the purpose of accelerating the data transmission rate and making the most of the crowded and limited spectral resource, some spectral efficient modulation formats, such as quadrature phase shift keying (QPSK) and quadrature amplitude modulation (QAM) [2], have been adopted. In these modulation formats, the amplitude of the RF signal is modulated, and the envelope of the RF signal is no longer constant and it is variable with large peak-to-average power ratio (PAPR), as shown in Fig. 2. In order to satisfy the stringent linearity requirement of the output RF signal, LPA is usually employed. If the LPA is powered by a constant supply voltage, it would suffer from very low efficiency since the voltage difference 


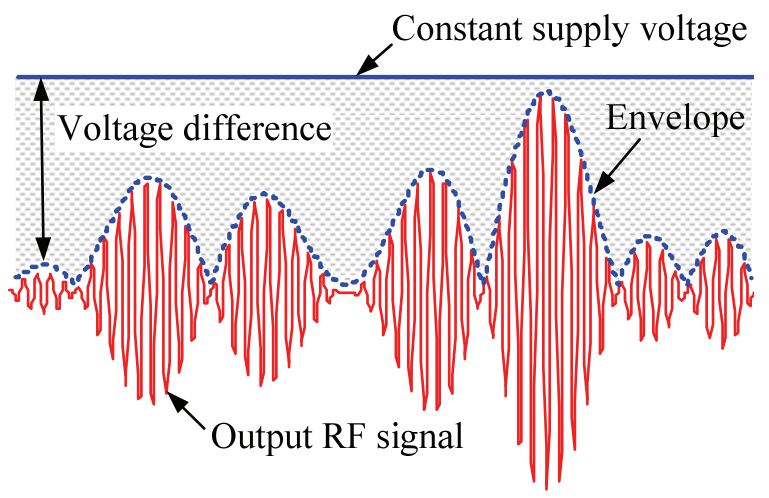

Fig. 2. Key waveforms of the LPA in $3 \mathrm{G}$ and $4 \mathrm{G}$ mobile communication systems.

between the supply voltage and output RF signal is very large.

Fig. 3 shows the curve of the drain efficiency (DE) versus the output power $P_{\text {out }}$ of a real class AB LPA when powered by a $28 \mathrm{~V}$ constant voltage [4]. As seen, when the LPA outputs $47 \mathrm{dBm}$ power, the DE reaches $65 \%$. However, the DE drops quickly with the output power backing off. For example, the DE falls to $10 \%$ when the LPA outputs $30 \mathrm{dBm}$ power. Fig. 3 also gives the power generation distribution (PGD) of the output RF signal with $8.5 \mathrm{~dB}$ PAPR when the LPA outputs a $40 \mathrm{dBm}$ average power. Obviously, the probability that the LPA works at its low efficiency region is large. As a result, the average efficiency of the LPA is about $30 \%$, and a large portion of energy is wasted.

In order to improve the efficiency of the LPA at the power back-off region, many techniques have been proposed. Among these techniques, Doherty technique [5]-[12], envelope elimination and restoration (EER) technique [13]-[20] and envelope tracking (ET) technique [21]-[28] are the most popular, and the ET technique is found to be the most promising for future mobile communication systems. The basic idea of the ET technique is to modulate the supply voltage of the LPA to track the envelope of the RF signal, and thus, the DE of the LPA is improved. Such power supply in the ET technique is usually called ET power supply. With the development of the mobile communication systems, the bandwidth and PAPR of the envelope signal is increasing, imposing stringent challenges for the design of the ET power supply.

As well known, switched-mode converter is featured with high efficiency compared with linear amplifier, and it is preferred to construct the ET power supply. However, when the bandwidth of the envelope signal is too high, the switchedmode converter should be operated at a very high switching frequency, resulting large switching loss and thus degrading the efficiency. As a result, the switching loss or the switching frequency needed to track the broadband envelope signal should be minimized for achieving a high efficiency.

The objectives of this paper are to reveal the essential reason why the LPA is less efficient in modern mobile communication systems, the superiorities of the ET technique, and how to build an ET power supply with high efficiency



Fig. 3. DE curve of the LPA when powered by $28 \mathrm{~V}$ constant voltage and PGD of the RF output signal versus the output power.

and wide bandwidth from the aspect of structure and control strategy. Our previous works [29], [30] have addressed these issues, and more detailed analyses are added in this paper. This paper is organized as follows. Section II presents the operating principle of the Doherty, EER and ET techniques, the pros and cons of each technique are analyzed, and it is pointed out that ET technique is the most promising technique of enhancing the efficiency of the LPA in future mobile communication systems. In Section III, the existing ET power supplies are classified into three types and detailed comparison is presented. In Section IV, the methods aiming for increasing the efficiency of the ET power supply are given, and the operating mechanisms are illustrated. Finally, Section $\mathrm{V}$ concludes this paper.

\section{EFFICIENCY ENHANCEMENT TECHNIQUES FOR POWER AMPLIFIER}

As illustrated above, the LPA supplied by constant voltage suffers from low efficiency when amplifying RF signals with large PAPR. Thus, advanced PA techniques have been proposed to achieve high efficiency and satisfy the linearity requirement simultaneously. In this section, the popular advanced PA techniques, including the Doherty technique, EER technique, and ET technique, will be discussed.

\section{A. Doherty Technique}

The Doherty technique was invented by William H. Doherty in 1936 [5]. Fig. 4 shows the schematic diagram of the even Doherty amplifier, which is composed of a splitter, a carrier amplifier, a peak amplifier, and three quarter-wave transmission lines (line 1, line 2, and line 3 ). The carrier amplifier is realized by a LPA to satisfy the linearity requirement of the output RF signal. In order to enhance the efficiency of the carrier amplifier at power back-off region, the peak amplifier, which is realized by a NLPA due to its high efficiency, and line 1 are employed to modulate the load resistance of the carrier amplifier by active load-pull technique. Since line 1 introduce $90^{\circ}$ phase shift to the output RF signal from the carrier amplifier, line 2 is added to the RF path of the peak amplifier to balance the phase shift. Meanwhile, line 3 is 


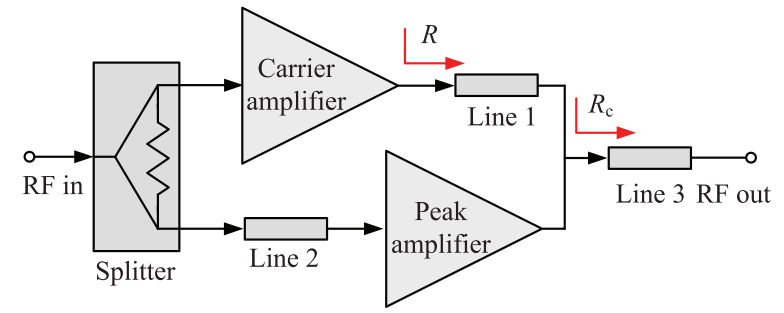

Fig. 4. Schematic diagram of the even Doherty amplifier.

added to realize impedance transformation because the output impedance of a typical RF system is $50 \mathrm{Ohm}$.

The RF input signal is split into two parts by the splitter. When the input power is low, only the carrier amplifier works, and the peak amplifier is shut down. While increasing the input power level to $6 \mathrm{~dB}$ power back-off from the maximum RF input power, carrier amplifier and peak amplifier work together.

Here, a class B power amplifier is taken as the carrier amplifier to explain the operating principle of the Doherty technique. Fig. 5 shows the circuit of class B power amplifier, where $C_{\text {block } 1}$ and $C_{\text {block } 2}$ are the dc blocking capacitors, $L_{\text {choke1 }}$ and $L_{\text {choke }}$ are the RF chokes, $v_{\mathrm{gs}}$ is the driving signal, $V_{\mathrm{th}}$ is the threshold voltage of the power amplifier, $i_{\mathrm{d}}$ is the drain current, $v_{\mathrm{ds}}$ is the drain voltage, $V_{\mathrm{DC}}$ is the dc power supply of the power amplifier, and $R$ is the load resistance of the power amplifier, as given in Fig. 4.

Fig. 6 shows the voltage and current waveforms and load lines of class B power amplifier. Since the power amplifier is biased at class $\mathrm{B}, i_{\mathrm{d}}$ is a half-rectified sine wave, and its fundamental component $I_{1}$ when the maximum drain current is $I_{\max }$, as shown in Fig. 6(a), can be expressed as

$$
I_{1}=I_{\max } / 2
$$

And the DC component $I_{0}$ of $i_{\mathrm{d}}$ can be expressed as

$$
I_{0}=I_{\max } / \pi
$$

The DE can be calculated by dividing the RF fundamental output power, $P_{1}$, by the input power from $V_{\mathrm{DC}}, P_{\mathrm{DC}}$. Thus, according to (1) and (2), the DE can be calculated by

$$
\mathrm{DE}=\frac{P_{1}}{P_{\mathrm{DC}}}=\frac{V_{1} \cdot I_{1}}{V_{\mathrm{DC}} \cdot I_{0}}=\frac{\frac{V_{\mathrm{DC}}}{\sqrt{2}} \cdot \frac{I_{\max }}{2 \sqrt{2}}}{V_{\mathrm{DC}} \cdot \frac{I_{\max }}{\pi}}=\frac{\pi}{4}
$$

It can be seen from (3) that, the full driven class B power amplifier, as shown in Fig. 6(a), can achieve $\pi / 4$ efficiency theoretically.

Fig. 6(b) shows the key waveforms of the class B power amplifier when the input RF power decreases $6 \mathrm{~dB}$ (onefourth of maximum power) when compared with Fig. 6(a). At this time, the peak current of $i_{\mathrm{d}}$ and voltage swing of $v_{\mathrm{ds}}$ are halved, and the $I_{1}$ and $I_{0}$ can be expressed as

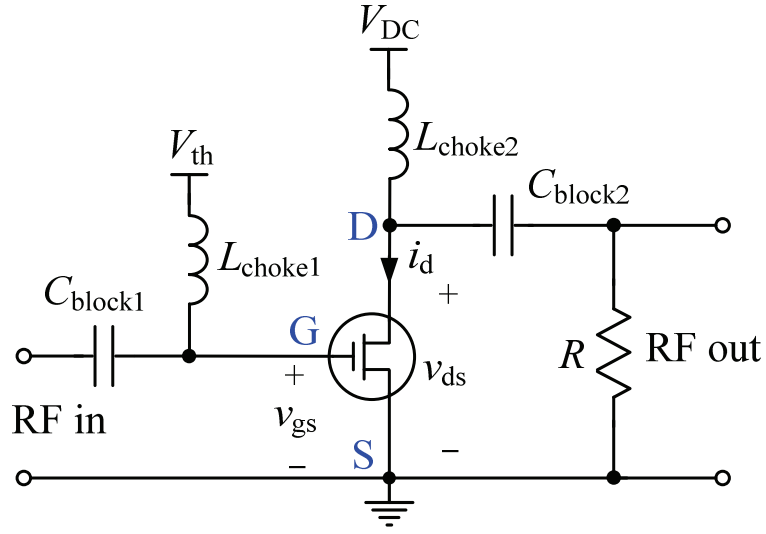

Fig. 5. Circuit of class B power amplifier.

$$
\begin{gathered}
I_{1}=I_{\text {max }} / 4 \\
I_{0}=\frac{I_{\max }}{2 \pi}
\end{gathered}
$$

According to (4) and (5), the DE can be expressed as

$$
\mathrm{DE}=\frac{P_{1}}{P_{\mathrm{DC}}}=\frac{V_{1} \cdot I_{1}}{V_{\mathrm{DC}} \cdot I_{0}}=\frac{\frac{V_{\mathrm{DC}}}{2 \sqrt{2}} \cdot \frac{I_{\max }}{4 \sqrt{2}}}{V_{\mathrm{DC}} \cdot \frac{I_{\max }}{2 \pi}}=\frac{\pi}{8}
$$

Comparing (3) and (6), it can be seen that the DE of the traditional class B power amplifier decreases with power backing off.

The load resistance of the class B power amplifier in Fig. 6(a) and (b) is $R$, while the load resistance is $2 R$ in Fig. 6(c). Comparing Fig. 6(b) and (c), the peak current of $i_{\mathrm{d}}$ is the same since the input power is identical. However, the voltage swing of $v_{\mathrm{ds}}$ in Fig. $6(\mathrm{c})$ is increased due to the increased load resistance. At this time, the DE can be expressed as

$$
\mathrm{DE}=\frac{P_{1}}{P_{\mathrm{DC}}}=\frac{V_{1} \cdot I_{1}}{V_{\mathrm{DC}} \cdot I_{0}}=\frac{\frac{V_{\mathrm{DC}}}{\sqrt{2}} \cdot \frac{I_{\max }}{4 \sqrt{2}}}{V_{\mathrm{DC}} \cdot \frac{I_{\max }}{2 \pi}}=\frac{\pi}{4}
$$

It can be seen from (7) that, when the input RF power is small, increasing the load resistance could increase the DE, as shown in Fig. 6(c), which is the mechanism of the Doherty technique. In conclusion, when the input RF power is small, the carrier amplifier should have high load resistance to achieve a high DE. However, the load resistance decreases with the increased input RF power. In order to adjust the load resistance according to the input RF power, the active load-pull technique is adopted.

Fig. 7 shows the simplified schematic diagram of the Doherty amplifier to illustrate the operating principle of the active load-pull technique, where CS1 and CS2 are two current sources, representing the carrier amplifier and peak amplifier, respectively, $R_{\mathrm{c}}$ is the common load of the carrier 


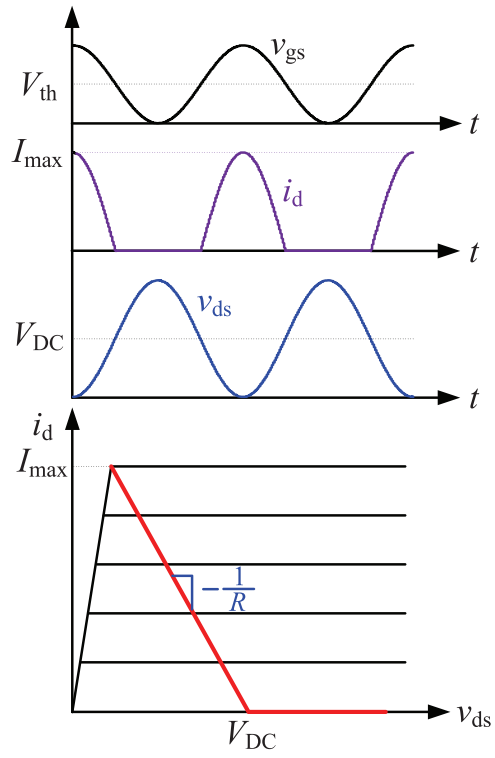

(a)

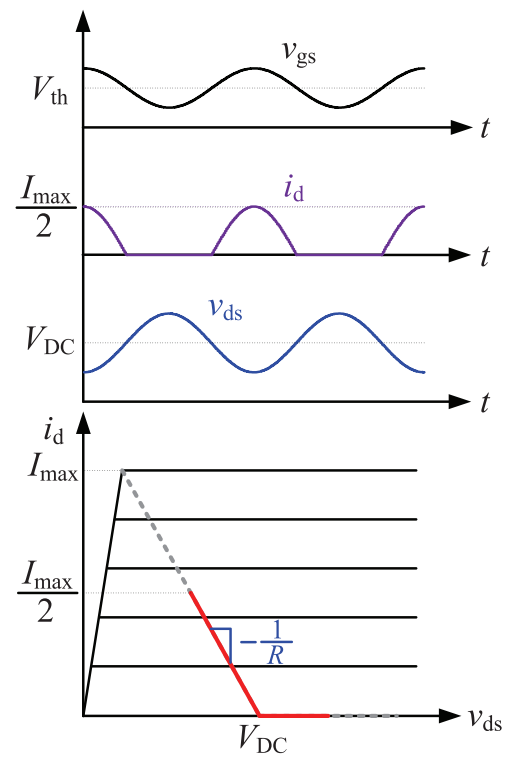

(b)

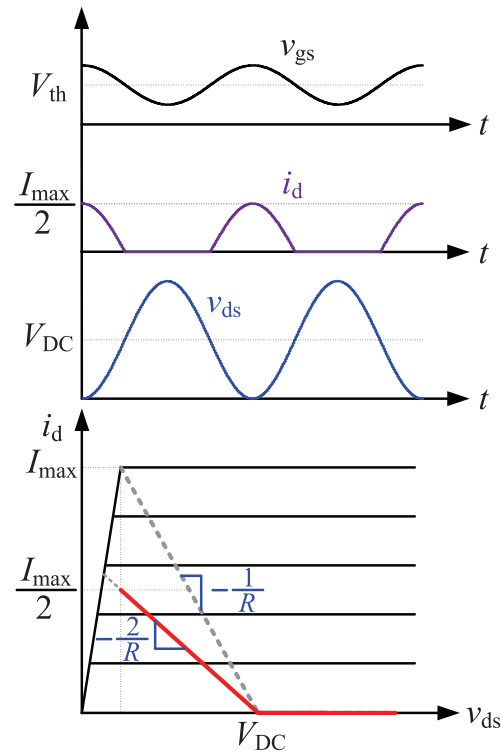

(c)

Fig. 6. Voltage and current waveforms and load lines of class B power amplifiers. (a) Full driven with load resistance $R$. (b) 6 dB back-off with load resistance $R$. (c) $6 \mathrm{~dB}$ back-off with load resistance $2 R$.

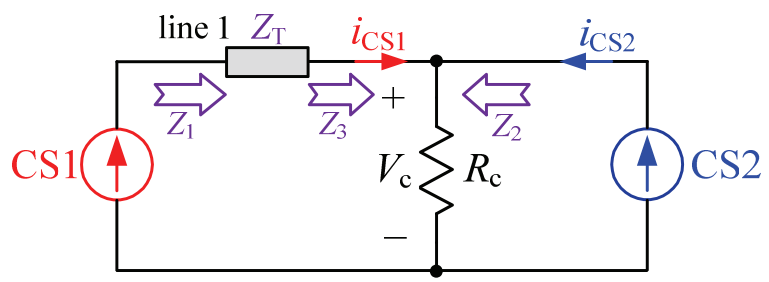

Fig. 7. Active load-pull technique.

amplifier and peak amplifier, as shown in Fig. 4 , and $Z_{\mathrm{T}}$ is the impedance of line1.

According to Fig. 7, $V_{\mathrm{c}}$ can be expressed as

$$
V_{\mathrm{c}}=R_{\mathrm{c}} \cdot\left(i_{\mathrm{CS} 1}+i_{\mathrm{CS} 2}\right)
$$

Thus, the resistance $Z_{3}$ can be expressed as

$$
Z_{3}=\frac{R_{\mathrm{c}} \cdot\left(i_{\mathrm{CS} 1}+i_{\mathrm{CS} 2}\right)}{i_{\mathrm{CS} 1}}=R_{\mathrm{c}} \cdot\left(1+\frac{i_{\mathrm{CS} 2}}{i_{\mathrm{CS} 1}}\right)
$$

Based on the characteristics of the quarter-wave transmission line [4], we have

$$
Z_{1}=\frac{Z_{T}^{2}}{Z_{3}}
$$

Thus, the load resistance of CS1, $Z_{1}$, can be adjusted by $i_{\mathrm{CS} 2}$, and $Z_{1}$ decreases with the increase of $i_{\mathrm{CS} 2}$.

\section{B. EER Technique}

EER technique was first invented by Leonard R. Kahn in 1952 [13] and received lots of attention ever since. Fig. 8 shows the schematic diagram and key waveforms of the EER tech-



Fig. 8. Schematic diagram and key waveforms of EER technique.

nique. The RF input signal, which includes both amplitude and phase information, is processed by two paths, namely, the envelope path and the phase path. In the envelope path, the envelope of the RF input signal is detected by the envelope detector and serves as the reference voltage of the supply modulator. The supply modulator outputs a voltage that tracks the envelope of the RF input signal and supplies the nonlinear power amplifier (NLPA). In the phase path, the envelope information of the RF input signal is eliminated by the limiter, producing a signal with constant amplitude and only containing the phase information of the RF input signal. The amplitude information is restored by the supply voltage provided by the supply modulator. The delay unit is used to synchronize the envelope path and the phase path.

Since a NLPA is adopted in EER technique, a high efficiency can be achieved. By introducing the supply modulator to modulate the supply voltage of the NLPA, the NLPA works like a high efficient LPA.

\section{ET Technique}

Fig. 9 shows the schematic diagram and key waveforms of the ET technique, which is similar to EER technique. The 


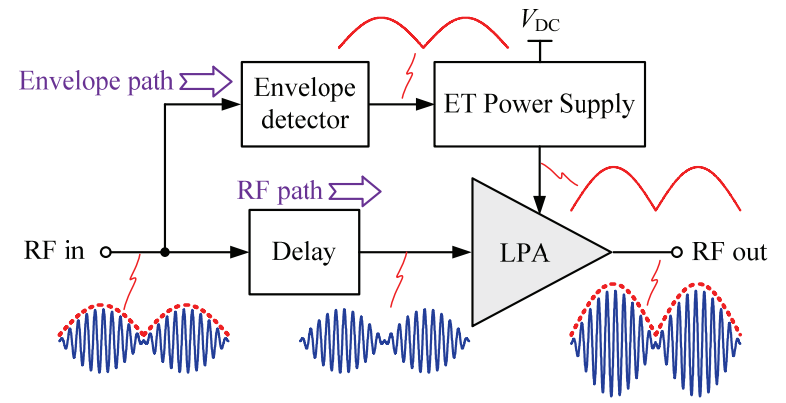

Fig. 9. Scheme diagram and key waveforms of ET technique.
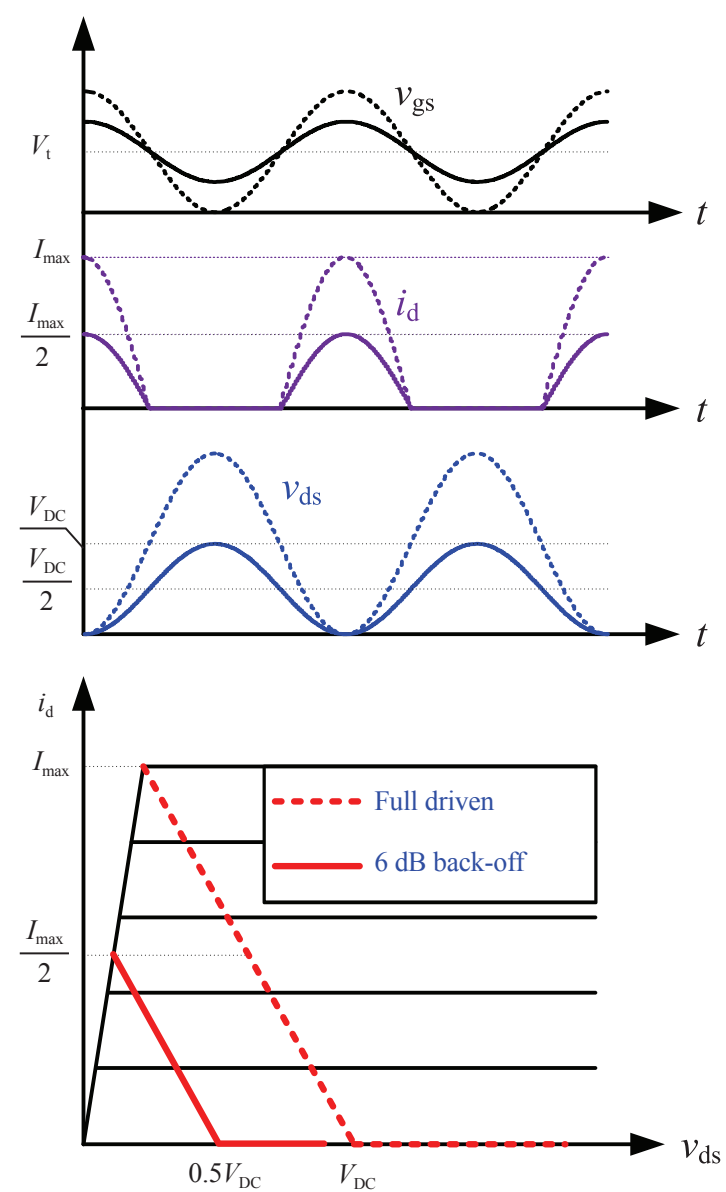

Fig. 10. Voltage and current waveforms and load lines of class B power amplifiers using ET technique at different power levels.

main differences between them can be summarized as: 1) a LPA is adopted in the ET technique, while a NLPA is used in the EER technique; 2) the RF signal amplified by the power amplifier includes both the amplitude and phase information in the ET technique, while the RF signal just contains phase information in EER technique; 3) The function of the supply modulator in EER technique is to restore the amplitude information of the output RF signal, while the ET power supply in ET technique aims for enhancing the efficiency of the LPA.

Fig. 10 shows the key waveforms of the class B power amplifier using ET technique at different power levels. When

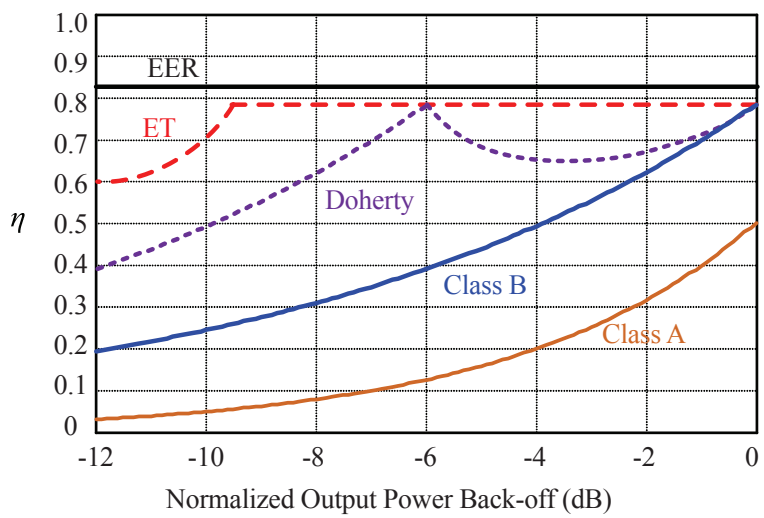

Fig. 11. Efficiency curves of power amplifiers using different techniques versus normalized output power back-off.

the class B power amplifier is full driven, as shown with the dotted lines, its efficiency can reach $\pi / 4$. When the input RF power backs off, the ET power supply outputs a lower voltage to supply the LPA correspondingly. The solid lines present the key waveforms of the class B power amplifier when the input power has a $6 \mathrm{~dB}$ back-off. At this time, the DE can be expressed as

$$
\mathrm{DE}=\frac{P_{1}}{P_{\mathrm{DC}}}=\frac{V_{1} \cdot I_{1}}{V_{\mathrm{DC}} \cdot I_{0}}=\frac{\frac{V_{\mathrm{DC}}}{2 \sqrt{2}} \cdot \frac{I_{\max }}{4 \sqrt{2}}}{\frac{V_{\mathrm{DC}}}{2} \cdot \frac{I_{\max }}{2 \pi}}=\frac{\pi}{4}
$$

From (11), the ET technique can also achieve a high DE at the power back-off region by dynamically adjusting the supply voltage of the LPA.

\section{Comparison of the Three Techniques}

Fig. 11 shows the efficiency curves of the amplifiers using different techniques, where the class $\mathrm{A}$ and class $\mathrm{AB}$ power amplifiers are powered by constant voltage. As seen, the Doherty, EER, and ET techniques are all capable of enhancing the efficiency of the power amplifier at the power backoff region. The power amplifier in the EER technique can achieve the highest efficiency, which could exceed $80 \%$ due to the adoption of NLPA. The LPA in ET technique could achieve $78.5 \%$ efficiency at high output power level when the LPA is biased at class B, and the efficiency decreases at low output power region due to the adoption of the envelope shaping [31]-[33], which results in the amplitude of the shaped envelope signal is higher than that of the original envelope signal. The efficiency curve of the Doherty amplifier is piecewise. At low output power region $(<-6 \mathrm{~dB})$, the peak amplifier shuts down, the carrier amplifier works with a large load resistance, leading to a higher efficiency than traditional class B power amplifier. At high output power region ( $>$ $-6 \mathrm{~dB})$, the carrier and peak amplifiers works together, and the efficiency keeps high.

Many mobile communication operators adopt Doherty technique in their base stations due to the simple structure. 
TABLE I

COMPARISON OF Doherty, EER, AND ET TeCHNIQUES

\begin{tabular}{ccc}
\hline \hline & Doherty & EER \\
\hline Power amplifier & LPA (Carrier amplifier) & NLPA \\
NLPA (Peak amplifier) & Medium & Highest \\
Efficiency & Narrow-band & Good \\
Linearity & Medium & Tighter \\
Delay matching & Medium & Tight \\
Requirement to & $/$ & High \\
supply modulator & $/$ & Medium \\
\hline \hline
\end{tabular}

However, the Doherty technique has several disadvantages: 1) it needs two power amplifiers, leading to increased cost; 2) at low output power region, the efficiency of the Doherty amplifier decreases quickly with output power backing off, which results in low efficiency when amplifying signals with very large PAPR; 3) it needs quarter-wave transmission lines to realize impedance transformation, which restricts its application in broadband application; and 4) since the peak amplifier is implemented with a NLPA, the linearity of the Doherty amplifier at high output power level is sacrificed. In conclusion, the Doherty amplifier is suitable for medium PAPR and narrow-band application, and can not meet the requirement of future mobile communication systems.

The EER technique could achieve the highest efficiency. However, EER technique also has some problems: 1) since the amplitude information is restored by the supply modulator, the linearity of the system completely lies on the linearity of the supply modulator, which imposes great challenges for the design of the supply modulator; 2) the linearity of the system is very sensitive to the delay matching between the envelope path and the phase path, and any mismatching between them will result in distortion of the RF output signal; 3) the EER system has a low gain in the low-power region and results in a poor linearity; and 4) the limiter will extend the bandwidth of the RF input signal, which poses challenges on the design of the broadband limiter and power amplifier. All of these issues limit its application.

Comparing with the EER technique, the linearity of the ET system is mainly determined by the LPA, thus the linearity requirement of the ET power supply are not so stringent, which makes the selection of its output voltage flexible. Thus, the requirement to the bandwidth of the ET power supply could be lowered. Meanwhile, the accuracy of the delay matching between the envelope path and RF path in ET technique could be decreased without bringing distortion to the RF output signal. These advantages make ET technique more suitable for broadband application. Besides, the ET technique has a higher gain in low output power region with envelope shaping, which avoids gain collapse and improves the linearity of the system.

TABLE I shows the detailed comparison of these three efficiency enhancement techniques, and the ET technique is
TABLE II

The PAPR and Bandwidth of the Envelope Signals for Different Mobile Communication Systems

\begin{tabular}{cccc}
\hline \hline \multicolumn{2}{c}{ Communication system } & PAPR $(\mathrm{dB})$ & Bandwidth $(\mathrm{MHz})$ \\
\hline $2 \mathrm{G}$ & $\mathrm{GSM}$ & 0 & \\
& & & \\
$2.5 \mathrm{G}$ & EDGE & 3.2 & 0.2 \\
& CDMA2000 & & 1.25 \\
$3 \mathrm{G}$ & WCDMA & $3.5 \sim 9$ & 5 \\
& TD-SCDMA & & 1.6 \\
$4 \mathrm{G}$ & LTE & $8.5 \sim 13$ & $2.4 \sim 20$ \\
\hline \hline
\end{tabular}

the most promising technique for future mobile communication systems due to easily implementation and suitable for broadband application.

\section{ET POWER SupPLIES}

ET power supply is the core of the ET technique and plays a crucial role in the efficiency of the ET system $\eta_{\mathrm{ET}}$. According to Fig. 9, $\eta_{\mathrm{ET}}$ can be approximated as

$$
\eta_{\mathrm{ET}}=\eta_{\mathrm{PS}} \cdot \eta_{\mathrm{LPA}}
$$

where, $\eta_{\mathrm{PS}}$ is the efficiency of the ET power supply, and $\eta_{\mathrm{LPA}}$ is the efficiency of the LPA.

In order to achieve a high $\eta_{\mathrm{ET}}$, the ET power supply is expected to achieve a high efficiency itself. Besides, the ET power supply should have a higher bandwidth than the envelope of the RF input signal so that its output voltage can well track the envelope to guarantee the LPA can achieve high efficiency. In conclusion, the ET power supply should achieve both wide bandwidth and high efficiency. TABLE II gives the PAPR and bandwidth of the envelope signal for different mobile communication systems. As seen, with the development of mobile communication systems, the PAPR and bandwidth of the envelope signal are increasing, imposing the challenges for designing the ET power supply.

Various structures of the ET power supply have been presented in previous publications, which can be classified into three categories, namely, linear amplifier structure [34], [35], 


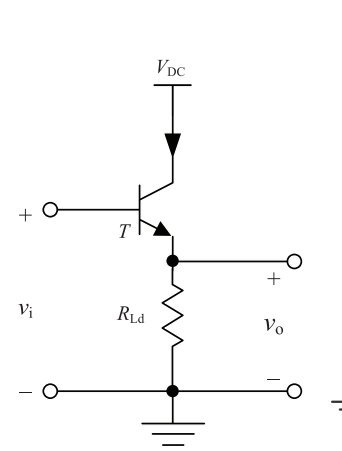

(a)

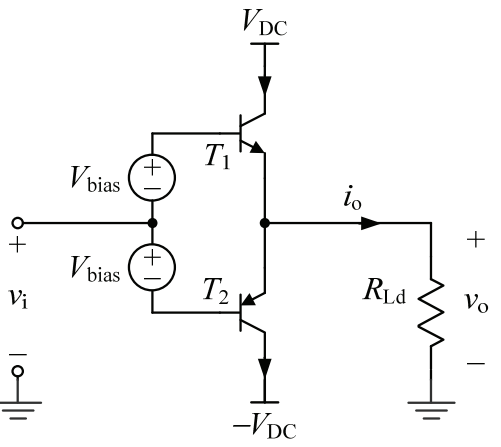

(b)
Fig. 12. Circuits of typical linear amplifiers. (a) Class A. (b) Class AB.

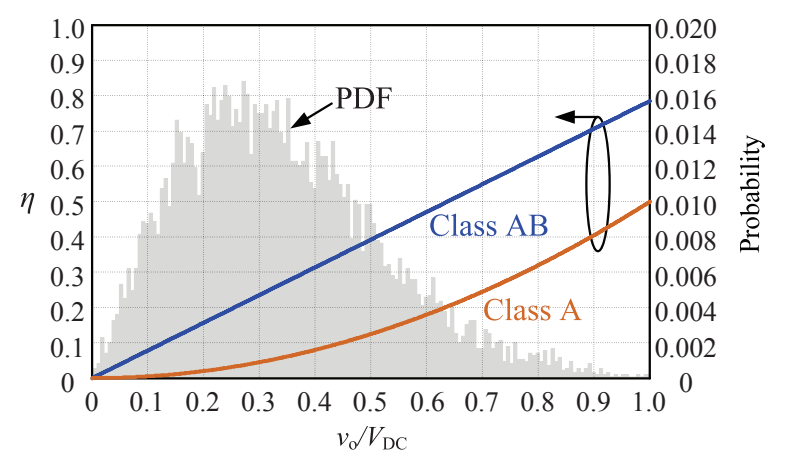

Fig. 13. Efficiency curves of the linear amplifiers and PDF of the envelope signal versus normalized output voltage.

switched-mode converter structure [36]-[42], and switch-linear hybrid (SLH) structure [43]-[50].

\section{A. Linear Amplifier Structure}

Fig. 12 shows the circuits of typical linear amplifiers, where the LPA is represented by $R_{\mathrm{Ld}}$ here since it can be equivalent to a constant resistor when supplied by the ET power supply [21]. Fig. 12(a) shows the circuit of class A linear amplifier, where $T$ is the power device. The class A linear amplifier can output unidirectional voltage and current, and its theoretical maximum efficiency is $50 \%$. Fig. 12(b) shows the circuit of the class AB linear amplifier, where $T_{1}$ and $T_{2}$ are the power devices, and $V_{\text {bias }}$ is the bias voltage to avoid crossover distortion. $T_{1}$ conducts in the positive part of the input signal, and $T_{2}$ conducts in the negative part. The class AB linear amplifier can output unidirectional voltage and bidirectional current, and its theoretical maximum efficiency is $78.5 \%$.

The linear amplifiers feature with high bandwidth and small output voltage ripple, and they have good ability of tracking the envelope signal, guaranteeing that the LPA achieves high efficiency and no spectrum disturbance occurs. Fig. 13 shows the efficiency curves of the linear amplifiers and probability density distribution (PDF) [28] of the envelope signal with $8.5 \mathrm{~dB}$ PAPR. It can be seen that the efficiencies of the linear amplifiers decrease with the output voltage. Referring to the PDF curve, there is a large probability that the envelope signal lies in the low amplitude

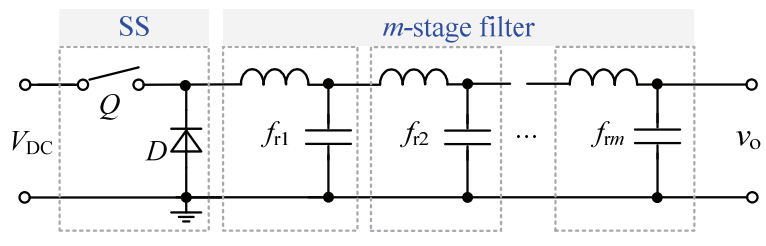

Fig. 14. Buck converter with $m$-stage filters.

region, where the linear amplifier is less efficient. More than that, the PDF would move left if the PAPR of the envelope signal becomes higher, and this would further worse the efficiency of the linear amplifiers. In future mobile communication systems, the PAPR will be continuously increased, and the ET power supply using the linear amplifier structure is not efficient.

\section{B. Switched-Mode Converter Structure}

The switched-mode converter can achieve high efficiency and it is preferred to construct the ET power supply. According to the number of power switches used, the switch network can be classified into single-switch (SS) network and multiple-switch (MS) network. Therefore, the switchedmode converter structure can be categorized as SS Structure and MS Structure. For the base station application, a stepdown converter is needed, and an open-loop controlled buck converter is usually employed to be a ET power supply or a basic cell due to its simple structure [39]-[41]. The following analysis is on the basis of the buck converter, and the analysis can be extended to other converters.

\section{1) SS Structure}

Fig. 14 shows the circuit of the buck converter with $m$-stage filter. Each stage filter is composed of an inductor and a capacitor, and the resonant frequency of each stage filters are $f_{\mathrm{r} 1}, f_{\mathrm{r} 2}, \ldots, f_{\mathrm{rm}}$, respectively.

When $m=1$, the circuit shown in Fig. 14 will be a traditional buck converter. For the purpose of attenuating the switching frequency component, the resonant frequency $f_{\mathrm{r} 1}$ should be far below the switching frequency $f_{\mathrm{s}}$, i.e., $f_{\mathrm{r} 1}<<f_{\mathrm{s}}$. The attenuation at the switching frequency, $\mathrm{A}_{1}\left(f_{\mathrm{s}}\right)$, can be expressed as

$$
\mathrm{A}_{1}\left(f_{\mathrm{s}}\right)=-40 \cdot \log \left(\frac{f_{\mathrm{s}}}{f_{\mathrm{r} 1}}\right)
$$

When the buck converter is open-loop controlled, its bandwidth $f_{\mathrm{BW}}$ approximately equals to $f_{\mathrm{r} 1}$, i.e., $f_{\mathrm{BW}} \approx f_{\mathrm{r} 1}$. Therefore, $f_{\mathrm{BW}}<<f_{\mathrm{s}}$. In order to track the envelope signal with wide bandwidth, the switching frequency of the SS structure should be very high. For example, if $f_{\mathrm{BW}}=20 \mathrm{MHz}, f_{\mathrm{s}}$ is required to be higher than $200 \mathrm{MHz}$. For such a high switching frequency, the traditional silicon-based power switches will suffer significant switching loss, greatly degrading the efficiency of the ET power supply. Fortunately, gallium nitride (GaN) high electron mobility transistors (HEMTs) emerged, which are suitable for operating at very high frequency with 


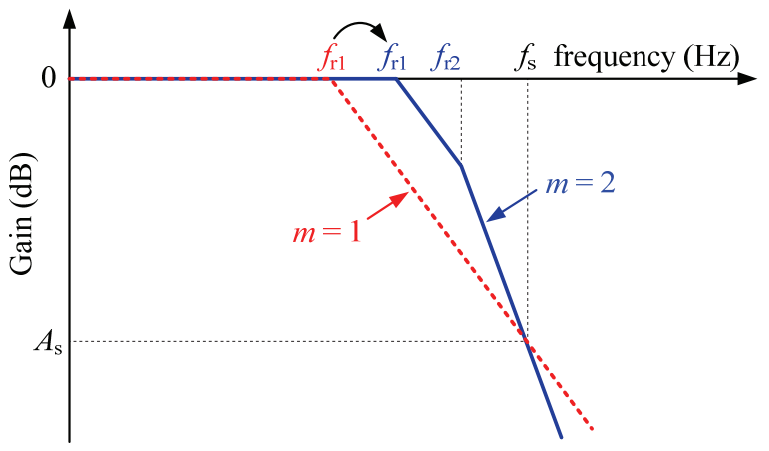

Fig. 15. Gain curves versus frequency of the single-stage and two-stage filters.

smaller switching loss [51]-[54]. When operating at such a high switching frequency, the parasitic parameters of the packaging and PCB layout have great impacts on the performance of the ET power supply. In order to effectively decrease the undesired parasitic parameters, the control circuits and the power devices are preferred to be integrated into a single chip [55], [56].

In order to track the envelope signal with reduced $f_{\mathrm{s}}, m$-stage filter $(m>1)$ can be used. At this time, the attenuation at the switching frequency $\mathrm{A}_{\mathrm{m}}\left(f_{\mathrm{s}}\right)$ can be expressed as

$\mathrm{A}_{m}\left(f_{\mathrm{s}}\right)=-40 \cdot \log \left(\frac{f_{\mathrm{s}}}{f_{\mathrm{r} 1}}\right)-40 \cdot \log \left(\frac{f_{\mathrm{s}}}{f_{\mathrm{r} 2}}\right)-\ldots-40 \cdot \log \left(\frac{f_{\mathrm{s}}}{f_{\mathrm{r} m}}\right)$

Fig. 15 shows the gain curves versus frequency of the single-stage and two-stage filters. It is clear to see that the minimum resonant frequency, $f_{\mathrm{r} 1}$, of the two-stage filters can be pushed closer to $f_{\mathrm{s}}$ for the same attenuation effect at $f_{\mathrm{s}}$, which is higher than that of the single-stage filter. Therefore, the bandwidth of the SS structure using $m$-stage filter $(m>1)$ can be increased. However, as $m$ increases, the design of the filter network is very complicated. Usually, $m=2$ is selected.

\section{2) MS Structure}

The purpose of the MS structure is to increase the equivalent switching frequency $f_{\mathrm{e} s}$. So, the frequency component needed to be suppressed is located at $n f_{\mathrm{s}}$, where $n$ is the number of switches used in the switch network. If the attenuation effect at $f_{\mathrm{e} s}$ is the same with the attenuation effect at $f_{\mathrm{s}}$ in SS structure, the resonant frequency of the filter in MS structure, $f_{\mathrm{e} \_\mathrm{r} 1}$, will be $n$ times $f_{\mathrm{r} 1}$. Also, when the MS structure is open-loop controlled, its bandwidth $f_{\mathrm{BW} \text { MS }}$ approximately equals to $f_{\mathrm{e}_{\mathrm{r} 1} 1}$. So, we have $f_{\mathrm{BW} \_\mathrm{MS}} \approx f_{\mathrm{e}_{\mathrm{r} 1} 1}=n f_{\mathrm{r} 1}$. This means that, compared with the SS structure, the bandwidth of MS structure, $f_{\mathrm{BW} \_\mathrm{MS}}$, can be increased by $n$ times.

Fig. 16 shows the schematic diagrams of typical MS structures, including the multilevel MS [44] and multiphase MS [54]. Fig. 16(a) shows a typical implementation of the multilevel MS structure, which is composed of a level provider and a level selector. The level provider generates a series of voltage levels $V_{1}, V_{2}, \ldots, V_{\mathrm{n}}\left(V_{1}<V_{2}<\ldots<V_{\mathrm{n}}=V_{\mathrm{DC}}\right)$. As all the voltage levels share the same ground, the level provider

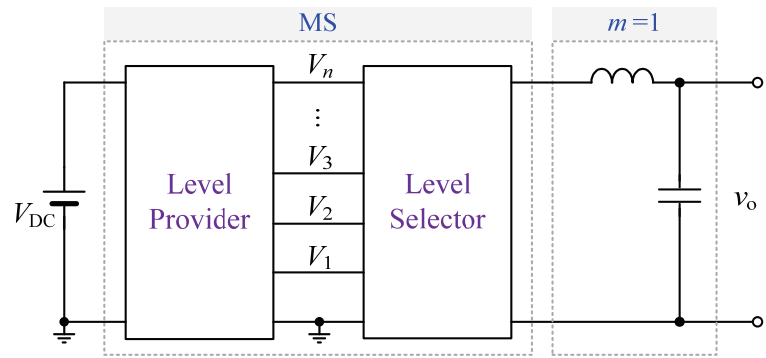

(a)

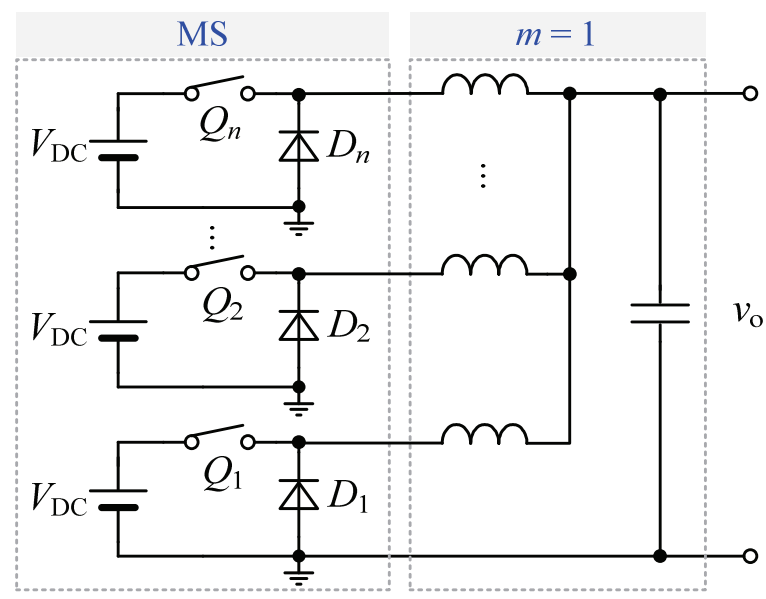

(b)

Fig. 16. Schematic diagrams of typical MS structures. (a) Multilevel MS. (b) Multiphase MS.



Fig. 17. PSD and PIC of the WCDMA envelope signal.

can be implemented by non-isolated dc-dc converters. The level selector chooses the corresponding voltage levels to roughly synthesize the envelope signal. Fig. 16(b) shows the schematic diagram of the multiphase MS structure. The phases are operated in an interleaved manner, thus the size of the filter network can be reduced due to the increased equivalent switching frequency and the ripple cancelation effect [49]. However, multiphase MS structure often requires a current-sharing loop to guarantee each phase providing equal power [49], which increases the system complexity and restricts the dynamic response. $m$-stage filter $(\mathrm{m}>1)$ can also be applied to MS structure to further improve the bandwidth. However, the system is very complex. 


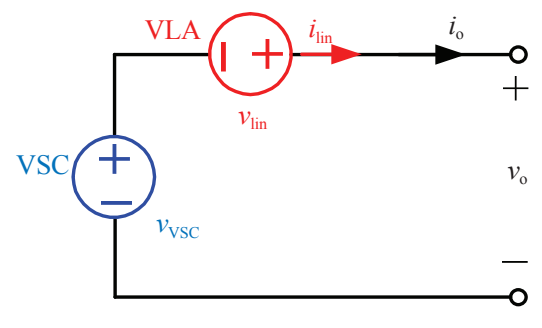

(a)

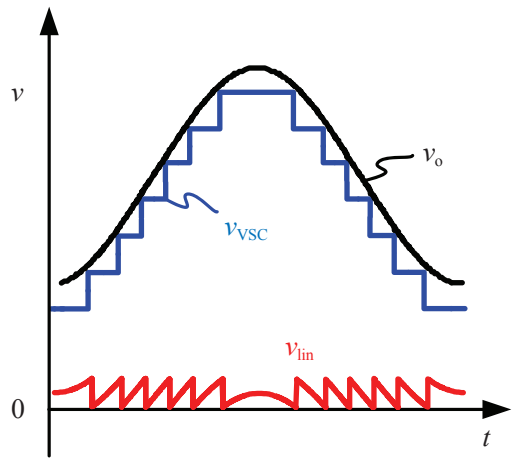

(b)

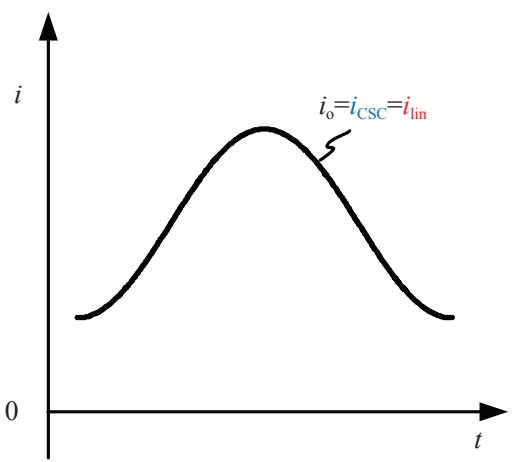

(c)

Fig. 18. Block diagram and key waveforms of series-form SLH ET power supply. (a) Block diagram. (b) Key voltage waveforms. (c) Key current waveforms.

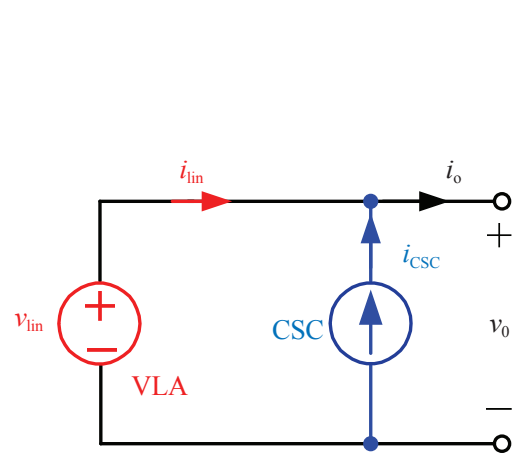

(a)



(b)



(c)

Fig. 19. Block diagram and key waveforms of parallel-form SLH ET power supply. (a) Block diagram. (b) Key voltage waveforms. (c) Key current waveforms.

\section{SLH Structure}

In order to track the envelope signal without distortion, the switching frequency or equivalent switching frequency of the switched-mode converter structure should be higher than the bandwidth of the envelope signal. Therefore, the required switching frequency to track a broadband envelope signal is still very high. Fig. 17 shows the power spectral density (PSD) and power integration curve (PIC) of the wideband code division multiple access (WCDMA) envelope signal. As seen, about $85 \%$ power is distributed from dc to $300 \mathrm{kHz}$ and $14 \%$ power lies between $300 \mathrm{kHz}$ to $5 \mathrm{MHz}$. Considering this power distribution, the SLH structure for ET power supply is proposed, in which, a switched-mode converter is used to provide the large portion low-frequency power with high efficiency, while a linear amplifier is employed to process the rest little portion high-frequency power. The SLH ET power supply integrates the advantages of the switchedmode converter and the linear amplifier, and it can achieve high efficiency and high bandwidth simultaneously. Since the switched-mode converter only processes the low-frequency components of the envelope signal, the switching frequency can be significantly reduced compared with that of switched-mode converter structure.
The SLH structure can be classified into series-form [57][59], parallel-form [60]-[62], and series-parallel-form [4], [48], [63].

\section{1) Series-Form $S L H$}

The series-form SLH ET power supply is composed of a voltage-controlled switched-mode converter (VSC) and a voltage-controlled linear amplifier (VLA), which are connected in series, as shown in Fig. 18(a). The VSC is usually implemented by a multilevel converter, as shown in Fig. 16(a), which outputs a step-wave voltage $v_{\mathrm{VSC}}$ to synthesize the load voltage $v_{0}$, and thus decreasing the output voltage swing of the linear amplifier, $v_{\text {lin }}$, as shown in Fig. 18(b). In doing so, the loss the linear amplifier is reduced, and thus the efficiency of the SLH ET power supply is greatly improved. However, the output current of the linear amplifier, $i_{\text {lin }}$, is still the load current $i_{\mathrm{o}}$, as shown in Fig. 18(c), which is large and still results in relatively large loss.

\section{2) Parallel-Form SLH}

The parallel-form SLH ET power supply is comprised of a current-controlled switched-mode converter (CSC) and a VLA, which are connected in parallel, as shown in Fig. 19(a). In this form, the CSC is expected to track $i_{\mathrm{o}}$, and the linear amplifier compensates the ripple current between $i_{\mathrm{o}}$ 
TABLE III

Comparison of the ET Power Supplies with Different Structures

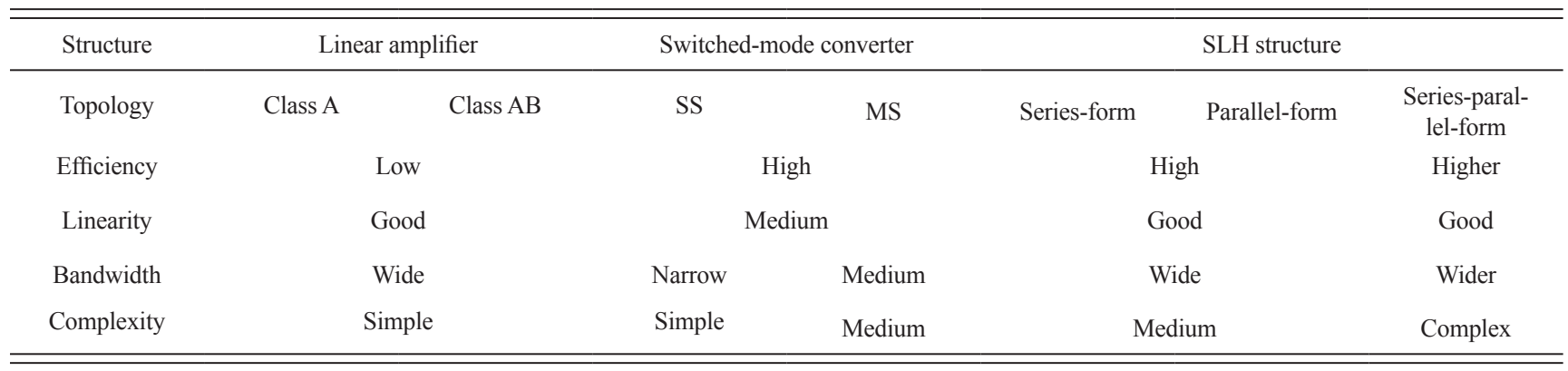

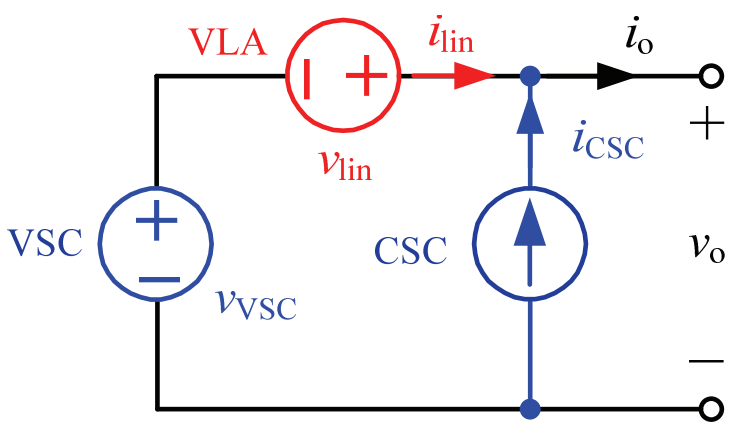

(a)

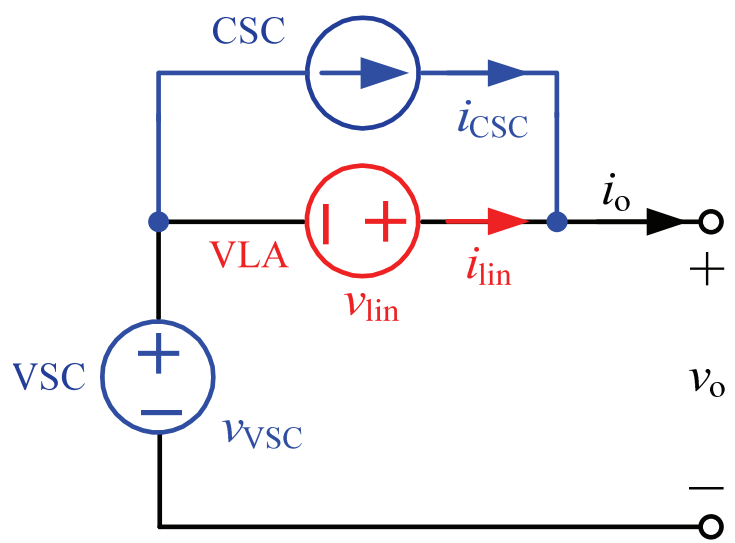

(b)

Fig. 20. Block diagram of series-parallel-form SLH ET power supply. (a) Type I. (b) Type II.

and the output current of CSC $i_{\mathrm{CSC}}$, as shown in Fig. 19(c). Thus, the loss of the linear amplifier is reduced, and the ET power supply can also achieve relatively high efficiency. However, $v_{\text {lin }}$ is equal to the load voltage $v_{0}$, as shown in Fig. 19(b), which still bring relatively large loss even if $i_{\text {lin }}$ is small.

\section{3) Series-Parallel-Form SLH}

Regardless of the series-form or the parallel-form, the linear amplifier still has relatively large loss, which hinders the increase of the efficiency of SLH ET power supply [64]. In order to further reduce the power loss of the linear amplifier, the series-parallel-form SLH ET power supply was proposed [48], which is composed by one linear amplifier and two

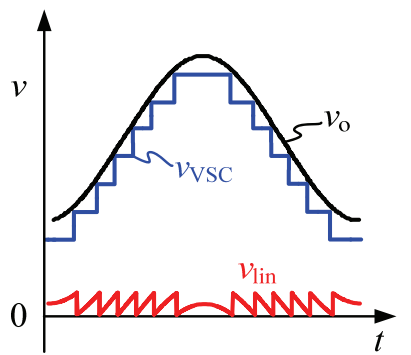

(a)

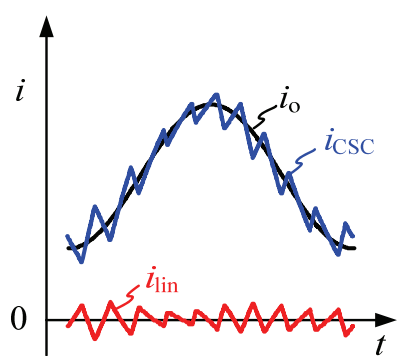

(b)
Fig. 21. Key waveforms of series-parallel-form SLH ET power supply. (a) Key voltage waveforms. (b) Key current waveforms.

switched-mode converters, which have two combinations, as shown in Fig. 20. As seen, one switched-mode converter is VSC, and the other is CSC. Fig. 21 shows the ideal voltage and current waveforms of the series-parallel-form SLH ET power supply. As seen, the $i_{\operatorname{lin}}$ and $v_{\operatorname{lin}}$ are both relatively small, leading to a reduced power loss in the linear amplifier.

TABLE III shows the comparison of the linear amplifier structure, the switched-mode converter structure, and SLH structure ET power supplies. As seen, the linear amplifier structure can achieve wide bandwidth and good linearity. However, its efficiency is relatively low. While the switchedmode converter structure features with higher efficiency and narrower bandwidth. The SLH structure combines the advantages of the linear amplifier structure and the switchedmode converter structure, and can achieve wide bandwidth and high efficiency simultaneously, and the series-parallel-form SLH structure can acquire higher efficiency and wider bandwidth due to the reduced power processed by the linear amplifier when compared with series-form and parallel-form SLH structures.

\section{Methods of Further Improving the EFficiency of ET POWER Supply}

In $4 \mathrm{G}$ mobile communication systems, the bandwidth of the envelope signal reaches $20 \mathrm{MHz}$, and it will be as high as $100 \mathrm{MHz}$ in $5 \mathrm{G}$ mobile communication systems. In order to track these broadband envelope signals, the switching frequency of the switched-mode converter in ET power supply should be very high, resulting large switching loss and 


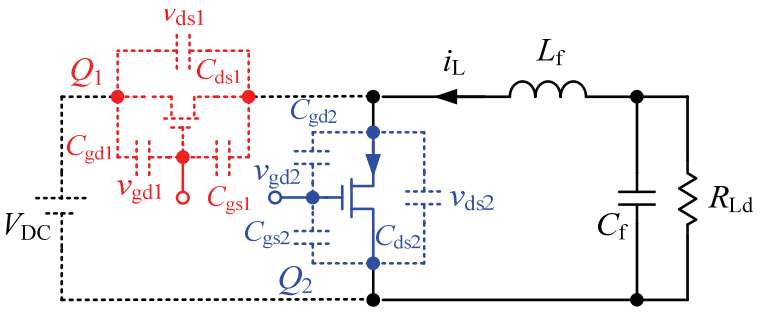

(a)

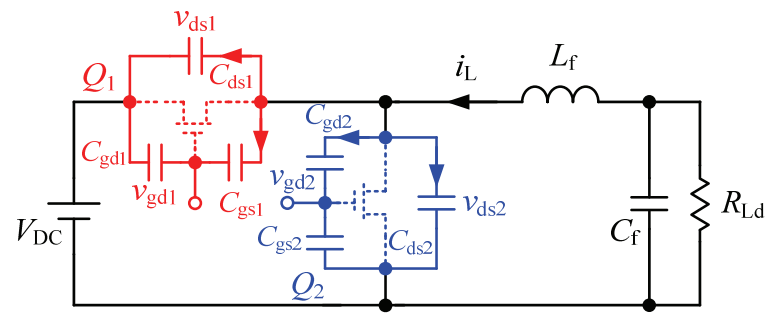

(b)

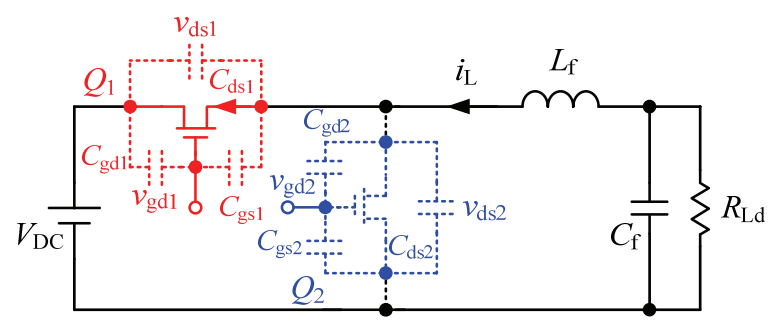

(c)

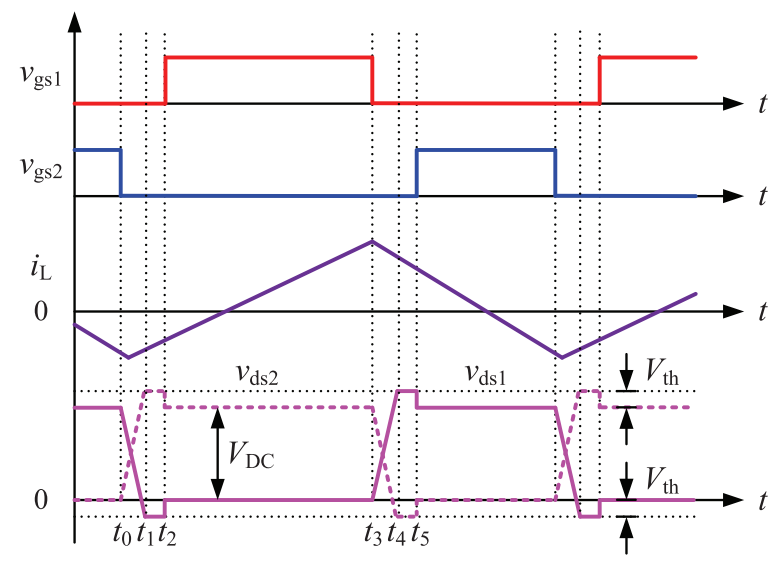

(d)

Fig. 22. Equivalent circuits and key waveforms of the buck converter. (a) Equivalent circuit before $t_{0}$. (b) Equivalent circuit during $\left[t_{0}, t_{1}\right]$. (c) Equivalent circuit during $\left[t_{1}, t_{2}\right]$. (d) Key waveforms.

thus degrading the efficiency. To achieve high efficiency, the switching loss or the switching frequency of the ET power supply should be reduced when tracking the broadband envelope signal.

\section{A. Soft-Switching Method}

To reduce the switching loss, GaN devices are preferred due to the reduced junction capacitors and fast switching frequency. Meanwhile, soft-switching method could be

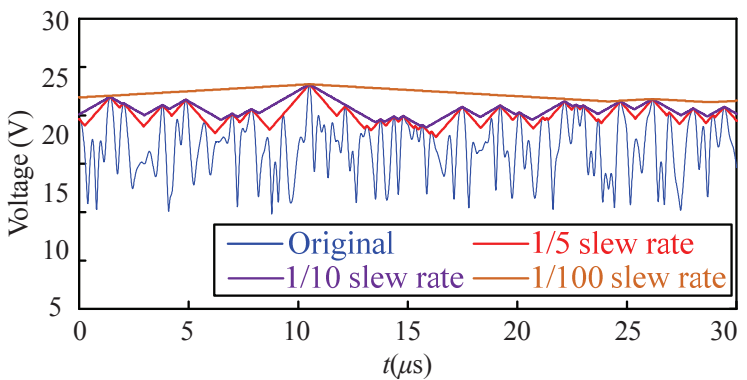

Fig. 23. Envelope signals with different slew rate.

employed. The simple buck converter is selected to explain the operating principle of the soft-switching method. Fig. 22 shows the equivalent circuits and key waveforms of the buck converter, where $Q_{1}$ and $Q_{2}$ are the power switches, $C_{\mathrm{ds} 1}$ and $C_{\mathrm{ds} 2}$ are the drain-source junction capacitors, $C_{\mathrm{gs} 1}$ and $C_{\mathrm{gs} 2}$ are the gate-source junction capacitors, $C_{\mathrm{gd} 1}$ and $C_{\mathrm{gd} 2}$ are the gate-drain junction capacitors, $v_{\mathrm{gs} 1}$ and $v_{\mathrm{gs} 2}$ are the driving signals of $Q_{1}$ and $Q_{2}$, respectively. Noted that here the filter inductor $L_{\mathrm{f}}$ is intentionally designed to be small enough so that its current could be negative. Prior to $t_{0}$, power switch $Q_{2}$ is conducting and power switch $Q_{1}$ is turned off, the inductor current $i_{\mathrm{L}}$ decays and crosses zero, as shown in Fig. 22(a). At $t_{0}$, power switch $Q_{2}$ is turned off, and the negative inductor current $i_{\mathrm{L}}$ charges $C_{\mathrm{gd} 2}$ and $C_{\mathrm{ds} 2}$, and discharges $C_{\mathrm{gd} 1}$ and $C_{\mathrm{ds} 1}$, thus, $v_{\mathrm{dg} 1}$ and $v_{\mathrm{ds} 1}\left(v_{\mathrm{dg} 1}=v_{\mathrm{ds} 1}, v_{\mathrm{dg} 1}=-v_{\mathrm{gd} 1}\right)$ decrease and cross zero, the equivalent circuit is shown in Fig. 22(b). At $t_{1}, v_{\mathrm{dg} 1}$ decays to $-V_{\text {th }}, Q_{1}$ conducts reversely, which provides approximate zero-voltage turn-on condition for $Q_{1}$, as shown in Fig. 22(c). The operating principle of the buck converter during $\left[t_{2}, t_{5}\right]$ is similar to that during $\left[0, t_{2}\right]$ and will not be explained here. As discussed above, both $Q_{1}$ and $Q_{2}$ realize zero-voltage-switching, and the switching loss is almost eliminated. The efficiency higher than $90 \%$ has been reported using GaN devices and soft-switching method [52], [53].

\section{B. Slow Envelope Method}

Since the switching loss is proportional to the switching frequency, the switching loss can be decreased by reducing the switching frequency. The slow envelope method has been proposed to lower the bandwidth of the original envelope signal [65]-[67]. By replacing the original envelope signal with the slow envelope signal as the reference voltage of the ET power supply, the bandwidth of the ET power supply can be decreased, indicating a reduced switching frequency. Thus, the efficiency of the ET power supply can be improved due to the reduced switching loss.

As reported in [65], several slow envelopes are generated, as shown in Fig. 23. Fig. 24 shows the PSD of the corresponding envelope signals, and it is clear to see that the bandwidth of the envelope signal can be greatly reduced by applying slow envelope method. Thus, the requirement to the ET power supply is relieved. However, the efficiency of the LPA is sacrificed. Therefore, there is a trade-off design between the efficiencies of the ET power supply and LPA. 


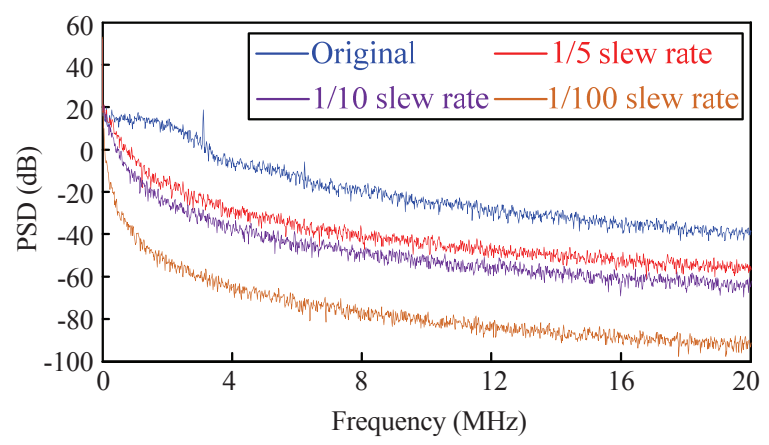

Fig. 24. Power spectral density of envelope signals with different slew rate.

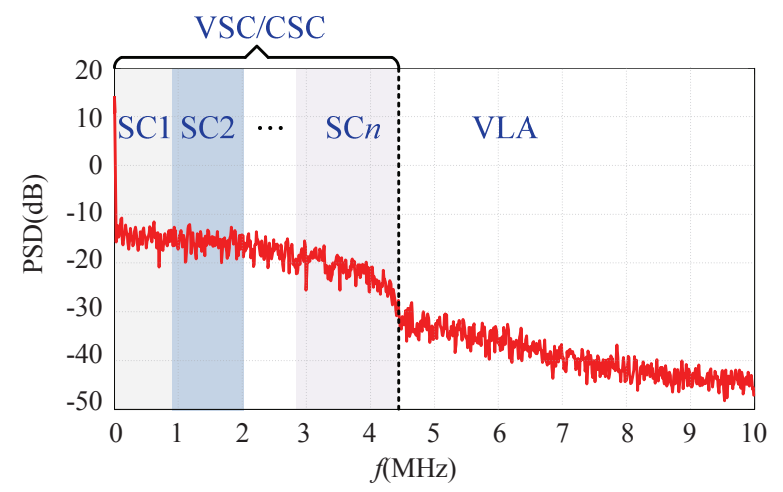

Fig. 25. Extension of band separation technique.

\section{Band Separation Method}

The envelope signal includes low frequency and high frequency components. In order to track the envelope signal accurately, the ET power supply should have the ability to track the high frequency components exactly, which will results in a waste of switching frequency when tracking the low frequency components. Thus, multiple converters with different tracking capability could be combined as an ET power supply to cope with different frequency components of the envelope signal, which is the basic thought of the band separation method.

The SLH structure is a representative example for applying the band separation method. The linear amplifier cooperates with the switched-mode converter to supply the frequency components of the envelope signal, i.e., the low-frequency components are processed by the switchedmode converter, while the high-frequency components are provided by the linear amplifier. The bandwidth of the switched-mode converter has a large impact on the overall efficiency. A lower bandwidth will increase the efficiency of the switched-mode converter, but the power processed by the VLA is increased with increased power loss. Therefore, the selection of the bandwidth of the switched-mode converter should trade off to achieve an overall efficiency of the ET power supply [68], [69].

Based on the SLH structure, the band separation method can be extended. Multiple switched-mode converters with different tracking capability, SC1, SC2, ..., and SCn,

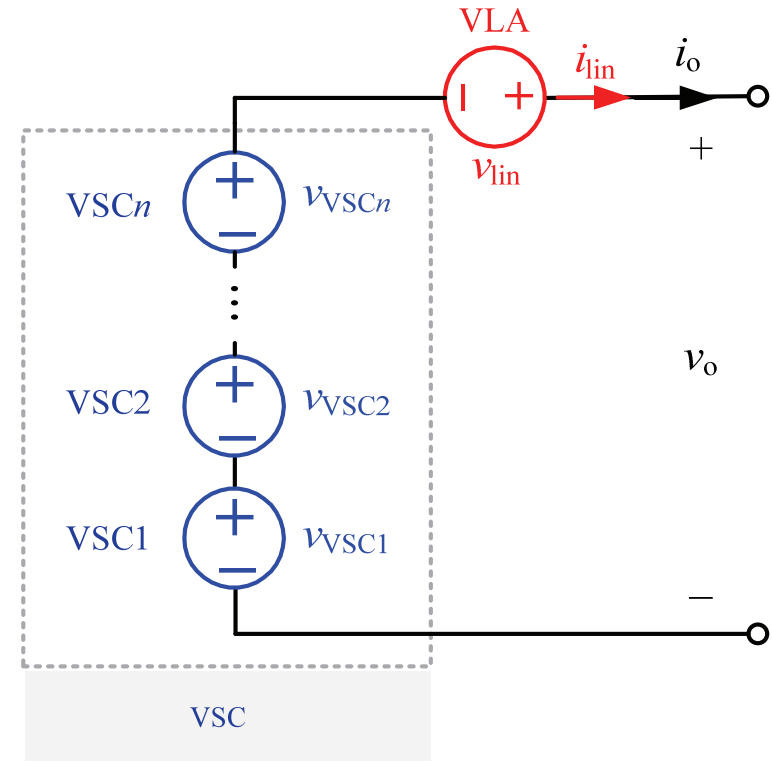

(a)

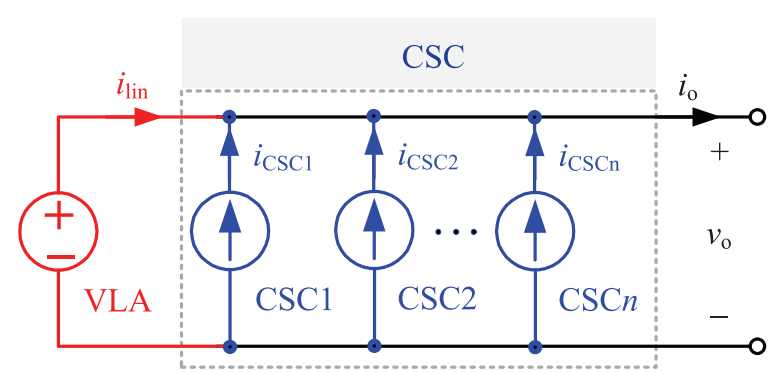

(b)

Fig. 26. SLH structures with multiple switched-mode converters. (a) Series-form SLH structure with multiple VSCs. (b) Parallel-form SLH structure with multiple CSCs.

can be employed, as shown in Fig. 25, and each switchedmode converter can be optimized independently. Compared to the SLH structure with single switched-mode converter, the SLH structure with multiple switched-mode converters can achieve a higher efficiency. Since the switched-mode converters can be either voltage-controlled or current-controlled, the basic SLH structure with multiple switchedmode converters has two combinations, as shown in Fig. 26. Besides, the band separation technique can also be extended into series-parallel-form SLH structure and switched-mode converter structure.

\section{Conclusion}

The mobile communication systems have been continuously developing to meet the demand of high data transmission rate. Thus, spectral efficient modulation formats have been employed, which are characterized with a variable envelope of the RF signal with large PAPR. The LPAs are adopted to satisfy the strict linearity requirement of the RF output signal. However, the LPAs powered by constant volt- 
age suffer from low efficiency. This paper introduces three popular efficiency enhancement techniques, i.e., Doherty, EER and ET techniques, to greatly improve the efficiency of the power amplifiers. The operating principle and characteristics of each technique are analyzed, and it is pointed out that the ET technique is the most suitable for future mobile communication systems. In the ET system, ET power supply is the core equipment and has a large impact on the system efficiency. The state-of-the-art ET power supplies are reviewed, and these ET power supplies are classified into the linear amplifier structure, the switched-mode converter structure, and the SLH structure. A comparative analysis is performed, which indicates that the SLH structure integrates the advantages of the former two structures and can achieve high efficiency and high bandwidth simultaneously. Finally, soft-switching method, slow envelope method, and band separation method, are presented aiming for further improving the efficiency of the ET power supply.

\section{REFERENCES}

[1] Z. Wang, Envelope tracking power amplifiers for wireless communications, Norwood, MA: Artech House, 2014.

[2] F. H. Raab, P. Asbeck, S. Cripps, P. B. Kenington, Z. B. Popovic N. Pothecary, J. F. Sevic, and N. O. Sokal, "Power amplifiers and transmitters for RF and microwave," IEEE Trans. Microw. Theory Tech., vol. 50, no. 3, pp. 814-826, Mar. 2002.

[3] S. C. Cripps, RF power amplifiers for wireless communications, Norwood, MA: Artech House, 2006.

[4] Y. Wang, X. Ruan, Q. Jin, Y. Leng, F. Meng, and X. -W. Zhu, "Quasi-interleaved current control for switch-linear hybrid envelope-tracking power supply," IEEE Trans. Power Electron., in press, 2017.

[5] W. H. Doherty, "A new high efficiency power amplifier for modulated waves," Proc. IRE., vol. 24, no. 9, pp. 1163-1182, Sept. 1936.

[6] F. H. Raab, "Efficiency of Doherty RF power-amplifier systems," IEEE Trans. Broadcast., vol. BC-33, no. 3, pp. 77-83, Sept. 1987.

[7] M. Iwamoto, A. Williams, P. Chen, A. G. Metzger, L. E. Larson, and P. M. Asbeck, "An extended Doherty amplifier with high efficiency over a wide power range," IEEE Trans. Microw. Theory Tech., vol.49, no. 12, pp. 2472-2479, Dec. 2001.

[8] B. Kim, J. Kim, I. Kim, and J. Cha, "The Doherty power amplifier," IEEE Microw. Mag., vol. 7, no. 5, pp. 42-50, Oct. 2006.

[9] H. Deguchi, N. Ui, K. Ebihara, K. Inoue, N. Yoshimura, and H. Takahashi, "A 33W GaN HEMT Doherty amplifier with 55\% drain efficiency for $2.6 \mathrm{GHz}$ base stations," IEEE MTT-S Int. Microw. Symp. Dig., pp. 1273-1276, Jun. 2009.

[10] Y. Yang, J. Cha, B. Shin, and B. Kim, "A fully matched N-way Doherty amplifier with optimized linearity," IEEE Trans. Microw. Theory Tech., vol. 51, no. 3, pp. 986-993, Mar. 2003.

[11] M. J. Pelk, W. C. E. Neo, J. R. Gajadharsing, R. S. Pengelly, and L. C. N. de Vreede, "A high-efficiency 100-W GaN three-way Doherty amplifier for base-station applications," IEEE Trans. Microw. Theory Tech., vol. 56, no. 7, pp. 1582-1591, Jul. 2008.

[12] Y. Yang, J. Cha, B. Shin, and B. Kim, "A microwave Doherty amplifier employing envelope tracking technique for high efficiency and linearity," IEEE Microw. Wireless Compon. Lett., vol. 13, no. 9, pp. 370-372, Sept. 2003.

[13] L. Kahn, "Single-sideband transmission by envelope elimination and restoration," Proc. IRE, vol. 40, no. 7, pp. 803-806, Jul. 1952.

[14] F. H. Raab, "Intermodulation distortion in Kahn-technique transmitters," IEEE Trans. Microw. Theory Tech., vol. 44, no. 12, pp. 22732278, Dec. 1996.

[15] T. Kwak, M. Lee, and G. Cho, "A 2 W CMOS hybrid switching amplitude modulator for EDGE polar transmitters," IEEE J. Solid-State Circuits, vol. 42, no. 12, pp. 2666-2676, Dec. 2007.
[16] D. Rudolph, "Kahn EER technique with single-carrier digital modulations," IEEE Trans. Microw. Theory Tech., vol. 51, no. 2, pp. 548-552, Feb. 2003.

[17] P. Reynaert and M. S. J. Steyaert, "A 1.75-GHz polar modulated CMOS RF power amplifier for GSM-EDGE," IEEE J. Solid-State Circuits, vol. 40, no. 12, pp. 2598-2608, Dec. 2005.

[18] J. Kitchen, W. Chu, I. Deligoz, S. Kiaei, and B. Bakkaloglu, "Combined linear and $\Delta$-modulated switched-mode PA supply modulator for polar transmitters," IEEE Int. Solid-State Circuits Conf. Tech. Dig., pp. 82-83, Feb. 2007.

[19] J. Choi, D. Kim, D. Kang, and B. Kim, "A polar transmitter with CMOS programmable hysteretic-controlled hybrid switching supply modulator for multistandard applications," IEEE Trans. Microw. Theory Tech., vol. 57, no. 7, pp. 1675-1686, Jul. 2009.

[20] F. Wang, D. Kimball, J. Popp, A. Yang, D. Lie, P. Asbeck, and L. Larson, "An improved power-added efficiency 19-dBm hybrid envelope elimination and restoration power amplifier for 802.11g WLAN applications," IEEE Trans. Microw. Theory Tech., vol. 54, no. 12, pp. 4086-4099, Dec. 2006.

[21] J. Moon, J. Son, J. Lee, and B. Kim, "A multimode/multiband envelope tracking transmitter with broadband saturated amplifier," IEEE Trans. Microw. Theory Tech., vol. 59, no. 12, pp. 3463-3473, Dec. 2011.

[22] F. Wang, A. H. Yang, D. F. Kimball, L. E. Larson, and P. M. Asbeck, "Design of wide-bandwidth envelope-tracking power amplifiers for OFDM applications," IEEE Trans. Microw. Theory Tech., vol. 53, no. 4, pp. 1244-1255, Apr. 2005.

[23] D. F. Kimball, J. Jeong, C. Hsia, P. Draxler, S. Lanfranco, W. Nagy, K. Linthicum, L. E. Larson, and P. M. Asbeck, "High-efficiency envelope tracking W-CDMA base station amplifier using GaN HFETs," IEEE Trans. Microw. Theory Tech., vol. 54, no. 11, pp. 3848-3856, Nov. 2006.

[24] Z. Wang, "Demystifying envelope tracking: Use for high-efficiency power amplifiers for 4G and beyond," IEEE Microw. Mag., vol. 16, no. 3, pp. 106-129, Mar. 2015

[25] M. Liu, D. Zhang, and Z. Zhou, "Linear regulator design considerations of the serial linear-assisted switching converter used as envelope amplifier," IEEE Trans. Power Electron., vol. 31, no. 5, pp. 3673-3689, Apr. 2016.

[26] P. F. Miaja, M. Rodriguez, A. Rodriguez, and J. Sebastian, “A linear assisted $\mathrm{dc} / \mathrm{dc}$ converter for envelope tracking and envelope elimination and restoration applications," IEEE Trans. Power Electron., vol. 27, no. 7, pp. 3302-3309, Jul. 2012.

[27] M. Vasic, O. Garcia, J. A. Oliver, P. Alou, D. Diaz, and J. A. Cobos, "Multilevel power supply for high-efficiency RF amplifiers," IEEE Trans. Power Electron., vol. 25, no. 4, pp. 1078-1089, Apr. 2010.

[28] Y. Wang, Q. Jin, and X. Ruan, "Optimized design of the multilevel converter in series-form switch-linear hybrid envelope tracking power supply," IEEE Trans. Ind. Electron., vol. 63, no. 9, pp. 5451-5460, Sep. 2016.

[29] X. Ruan, Q. Jin, "A review of envelope tracking power supply," Trans. China Electrotechnical Society., vol. 32, no. 4, pp. 1-11, Feb. 2017.

[30] H. Xi, X. Xiong, and X. Ruan, "Development and design challenges of high speed envelope tracking power supply," Trans. China Electrotechnical Society., vol. 26, no. 9, pp. 151-159, Sep. 2011.

[31] D. Kim, D. Kang, J. Choi, J. Kim, Y. Cho, and B. Kim, "Optimization for envelope shaped operation of envelope tracking power amplifier," IEEE Trans. Microw. Theory Tech., vol. 59, no. 7, pp. 1787-1795, July. 2011.

[32] J. Jeong, D. F. Kimball, M. Kwak, P. Draxler, and P. M. Asbeck, "Envelope tracking power amplifiers with reduced peak-to-average power ratio RF input signals," in Proc. IEEE Radio Wireless Symp., 2010: $112-115$.

[33] R. Wu, Y.-T. Liu, J. Lopez, C. Schecht, Y. Li, and D. Y. C. Lie, "High-efficiency silicon-based envelope-tracking power amplifier design with envelope shaping for broadband wireless applications," IEEE J. Solid-State Circuits., vol. 48, no. 9, pp. 2030-2040, Sept. 2013.

[34] J. Choi, D. Kim, D. Kang, M. Jun, B. Jin, J. Park, and B. Kim, “A 45/46/34\% PAE linear polar transmitter for EDGE/WCDMA/Mobile 
WiMax," IEEE MTT-S Intl. Microw. Symp. Dig., pp. 413-416, Jun. 2009.

[35] J. Hoversten, S. Schafer, M. Roberg, M. Norris, D. Maksimovic, and Z. Popovic, "Codesign of PA, supply, and signal processing for linear supply-modulated RF transmitter," IEEE Trans. Microw. Theory Tech., vol. 60, no. 6, pp. 2010-2020, Jun. 2012.

[36] J. Sankman, M. K. Song, and D. Ma, "Switching-converter-only multiphase envelope modulator with slew rate enhancer for LTE power amplifier applications," IEEE Trans. Power Electron., vol. 31, no. 1, pp. 817-826, Jan. 2016.

[37] M. Rodriguez, P. F. Miaja, A. Rodriguez, and J. Sebastian, "A multiple-input digitally controlled buck converter for envelope tracking applications in radiofrequency power amplifiers," IEEE Trans. Power Electron., vol. 25, no. 2, pp. 369-381, Feb. 2010.

[38] P. F. Miaja, A. Rodriguez, and J. Sebastian, "Buck-derived converters based on gallium nitride devices for envelope tracking applications," IEEE Trans. Power Electron., vol. 30, no. 4, pp. 2084-2095, Apr. 2015.

[39] S. Shinjo, Y. Hong, H. Gheidi, D. F. kimball, and P. M. Asbeck, "High speed, high analog bandwidth buck converter using GaN HEMTs for envelope tracking power amplifier applications," in Proc. IEEE WSSN., 2013: 13-15.

[40] Y. Hong, K. Mukai, H. Gheidi, S. Shinjo, and P. M. Asbeck, "High efficiency GaN switching converter IC with bootstrap driver for envelope tracking applications," in Proc. IEEE RFIC., 2013: 353-356.

[41] V. Mehrotra, A. Arias, J. Bergman, C. Neft, M. Urteaga, and B. Brar, "865 MHz switching-speed step-down DC-DC power converter for envelope tracking," in Proc. IEEE APEC., 2016: 79-85.

[42] V. Yousefzadeh, E. Alarcon, and D. Maksimovic, "Three-level buck converter for envelope tracking applications," IEEE Trans. Power Electron., vol. 21, no. 2, pp. 549-552, Mar. 2006.

[43] D. Diaz, M. Vasic, O. Garcia, J. A. Oliver, P. Alou, R. Prieto, and J. A. Cobos, "Three-level cell topology for a multilevel power supply to achieve high efficiency envelope amplifier," IEEE Trans. Circuits and Systems-I: Regular Papers, vol. 59, no. 9, pp. 1078-1089, Sept. 2012.

[44] M. Vasic, P. Cheng, O. Garcia, J. A. Oliver, P. Alou, J. A. Cobos, D. Tena, F. J. Ortega-Gonzalez, "The design of a multilevel envelope tracking amplifier based on a multiphase buck converter," IEEE Trans. Power Electron., vol. 31, no. 6, pp. 4611-4627, Jun. 2016.

[45] Q. Jin, X. Ruan, X. Ren, and H. Xi, "High-efficiency switch-linear-hybrid envelope-tracking power supply with step-wave approach," IEEE Trans. Ind. Electron., vol. 62, no. 9, pp. 5411-5421, Sept. 2015.

[46] D. kimball, T. Nakatani, J. Yan, P. T. Theilmann, and I. Telleiz, "High efficiency envelope tracking power amplifiers for wide modulation bandwidth signals (invited)," in Proc. APMC, 2014: 103-106.

[47] D. F. Kimball, J. Jinho, H. Chin, P. Draxler, S. Lanfranco, W. Nagy, K. Linthicum, L. E. Larson, and P. M. Asbeck, "High-efficiency envelope-tracking WCDMA base-station amplifier using GaN HFETs," IEEE Trans. Microw. Theory Tech., vol. 54, no. 11, pp. 3848-3856, Nov. 2006.

[48] Q. Jin, X. Ruan, X. Ren, Y. Wang, Y. Leng, and C. K. Tse, "Series-parallel form switch-linear hybrid envelope-tracking power supply to achieve high efficiency," IEEE Trans. Ind. Electron., vol. 64, no. 1, pp. 244-252, Jan. 2017.

[49] P. Y. Wu and P. K. T. Mok, "A two-phase switching hybrid supply modulator for RF power amplifiers with 9\% efficiency improvement," IEEE J. Solid-State Circuits, vol. 45, no. 12, pp. 2543-2556, Dec. 2010.

[50] M. Tan and W.-H. Ki, "An efficiency-enhanced hybrid supply modulator with single-capacitor current-integration control," IEEE J. Solid-State Circuits, vol. 51, no. 2, pp. 533-542, Feb. 2016.

[51] Y. Zhang, M. Rodriguez, and D. Maksimovic, "Very high frequency PWM buck converters using monolithic GaN half-bridge power stages with integrated gate drivers," IEEE Trans. Power Electron., vol. 31, no. 11, pp. 7926-7942, Jun. 2016.

[52] Y. Zhang, M. Rodriguez, and D. Maksimovic, " $100 \mathrm{MHz}, 20 \mathrm{~V}, 90 \%$ efficient synchronous buck converter with integrated gate driver," in Proc. IEEE ECCE, 2014: 3664-3671.

[53] Y. Zhang, M. Rodriguez, and D. Maksimovic, "High frequency synchronous buck converter using GaN-on-SiC HEMTs," in Proc. IEEE ECCE, 2013: 488-494.
[54] Y. Zhang, M. Rodriguez, and D. Maksimovic, "Output filter design in high-efficiency wide-bandwidth multi-phase buck envelope amplifiers," in Proc. IEEE APEC, 2015: 2026-2032.

[55] M. Kwak, J. Jeong, M. Hassan, J. J. Yan, D. F. Kimball, M. Asbeck, and L. E. Larson, "High efficiency wideband envelope tracking power amplifier with direct current sensing for LTE applications," in Proc. IEEE PAWR, 2012: 41-44.

[56] P. Asbeck, L. Larson, D. Kimball, M. Hassan, C. Hsia, C. Presti, and A. Scuderi, "Si IC development for high efficiency envelope tracking power amplifiers," in Proc. IEEE SiRF, 2012: 1-4.

[57] M. C. Gonzalez, M. Vasic, P. Alou, O. Garcia, J. A. Oliver, and J. A. Cobos, "Transformer-coupled converter for voltage modulation techniques," IEEE Trans. Power Electron., vol. 28, no. 5, pp. 2330-2342, May. 2013.

[58] M. Vasic, O. Garcia, J. A. Oliver, P. Alou, D. Diaz, R. Prieto, and J. A. Cobos, "Envelope amplifier based on switching capacitors for high-efficiency RF amplifiers," IEEE Trans. Power Electron., vol. 27, no. 3, pp. 1359-1368, Mar. 2012.

[59] P. Cheng, M. Vasic, O. Garcia, J. A. Oliver, P. Alou, and J. A. Cobos, "Minimum time control for multiphase buck converter: analysis and application," IEEE Trans. Power Electron., vol. 29, no. 2, pp. 958967, Feb. 2014

[60] H. Chin, Z. Anding, J. J. Yan, P. Draxler, D. F. Kimball, S. Lanfranco, and P. M. Asbeck, "Digitally assisted dual-switch high-efficiency envelope amplifier for envelope-tracking base-station power amplifiers," IEEE Trans. Microwave Theory Tech., vol. 59, no. 11, pp. 2943-2952, Nov. 2011.

[61] H. Xi, Q. Jin, X. Ruan, and X. Xiong, "Full feed-forward of the output voltage to improve efficiency for envelope-tracking power supply using switch-linear hybrid configuration," IEEE Trans. Power Electron., vol. 28, no. 1, pp. 451-456, Jan. 2013.

[62] P. F. Miaja, J. Sebastian, R. Marante, and J. A. cobos, "A linear assisted switching envelope amplifier for an UHF Polar Transmitter," IEEE Trans. Power Electron., vol. 29, no. 4, pp. 1850-1861, Apr. 2014.

[63] M. Hassan, L. E. Larson, V. W. Leung, and P. M. Asbeck, "A combined series-parallel hybrid envelope amplifier for envelope tracking mobile terminal RF power amplifier applications," IEEE J. Solid-State Circuits, vol. 47, no. 5, pp. 1185-1198, May. 2012.

[64] M. Vasic, O. Garcia, J. A. Oliver, P. Alou, and J. A. Cobos, "Theoretical efficiency limits of a serial and parallel linear-assisted switching converter as an envelope amplifier," IEEE Trans. Power Electron., vol. 29, no. 2, pp. 719-728, Feb. 2014.

[65] P. M. Cheng, O. Garcia, M. Vasic, P. Alou, J. A. Oliver, G. Montoro, and J. A. Cobos, "Envelope amplifier based on a hybrid series converter with the slow-envelope technique," in Proc. IEEE ECCE, 2012: $1-5$.

[66] G. Montoro, P. L. Gliabert, E. Bertran, and J. Berenguer, "A method for real-time generation of slew-rate limited envelopes in envelope tracking transmitters," in Proc. IEEE IMWS, 2010: 1-4.

[67] G. Montoro, P. L. Gliabert, P. Vizarreta, and E. Bertran, "Slew-rate limited envelopes for driving envelope tracking amplifiers," in Proc. IEEE PAWR, 2011: 17-20.

[68] V. Yousefzadeh, E. Alarcon, and D. Maksimovic, "Band separation and efficiency optimization in linear-assisted switching power amplifiers," in Proc. IEEE PESC, 2006: 1-7.

[69] D. Li, Y. Zhang, M. Roduiguez, and D. Maksimovic, "Band separation in linear-assisted switching power amplifiers for accurate wide-bandwidth envelope tracking," in Proc. IEEE ECCE, 2014: 1113-1118. 


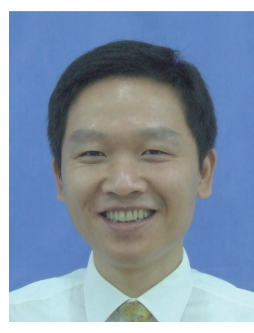

Xinbo Ruan received the B.S. and Ph.D. degrees in electrical engineering from Nanjing University of Aeronautics and Astronautics (NUAA), Nanjing, China, in 1991 and 1996, respectively.

In 1996, he joined the Faculty of Electrical Engineering Teaching and Research Division, NUAA, where he became a Professor in the College of Automation Engineering in 2002 and has been engaged in teaching and research in the field of power electronics. From August to October 2007, he was a Research Fellow in the Department of Electronic and Information Engineering, Hong Kong Polytechnic University, Hong Kong, China. Since March 2008, he has been also with the School of Electrical and Electronic Engineering, Huazhong University of Science and Technology, China. He is a Guest Professor with Beijing Jiaotong University, Beijing, China, Hefei University of Technology, Hefei, China, and Wuhan University, Wuhan, China. He is the author or co-author of 9 books and more than 200 technical papers published in journals and conferences. His main research interests include soft-switching dc-dc converters, soft-switching inverters, power factor correction converters, modeling the converters, power electronics system integration and renewable energy generation system.

Prof. Ruan was a recipient of the Delta Scholarship by the Delta Environment and Education Fund in 2003 and was a recipient of the Special Appointed Professor of the Chang Jiang Scholars Program by the Ministry of Education, China, in 2007. From 2005 to 2013, he served as Vice President of the China Power Supply Society, and since 2008, he has been a member of the Technical Committee on Renewable Energy Systems within the IEEE Industrial Electronics Society. Currently, he serves as an Associate Editor for the IEEE Transactions on Industrial Electronics, the IEEE Transactions on Power Electronics, the IEEE Journal of Emerging and Selected Topics on Power Electronics, and the IEEE Transactions on Circuits and Systems II. He is an IEEE Fellow.

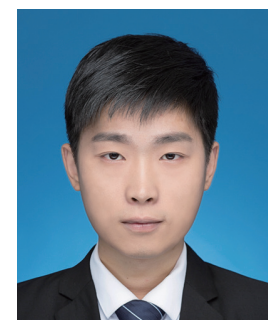

Yazhou Wang received the B. S. degree in electrical engineering and automation from Nanjing University of Aeronautics and Astronautics (NUAA), Nanjing, China, in 2013, where he is currently working toward the Ph.D. degree in electrical engineering.

His current research interests include envelope tracking power supplies and dc-dc converters.

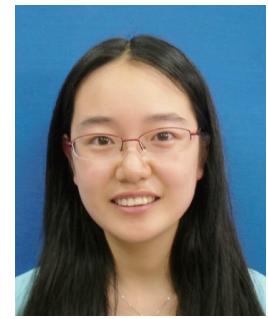

Qian Jin received the B. S. and Ph.D. degrees in electrical engineering and automation from Nanjing University of Aeronautics and Astronautics (NUAA), Nanjing, China, in 2011 and 2017, respectively.

Her current research interests include envelope tracking power supplies and dc-dc converters. 